\title{
Sollten Diabetiker frühstücken?
}

\author{
Verzichten Diabetiker aufs Frühstück, wirkt sich das negativ \\ auf die postprandiale Glukoseantwort bei späteren Mahlzei- \\ ten aus - so die Ergebnisse einer Studie.
}

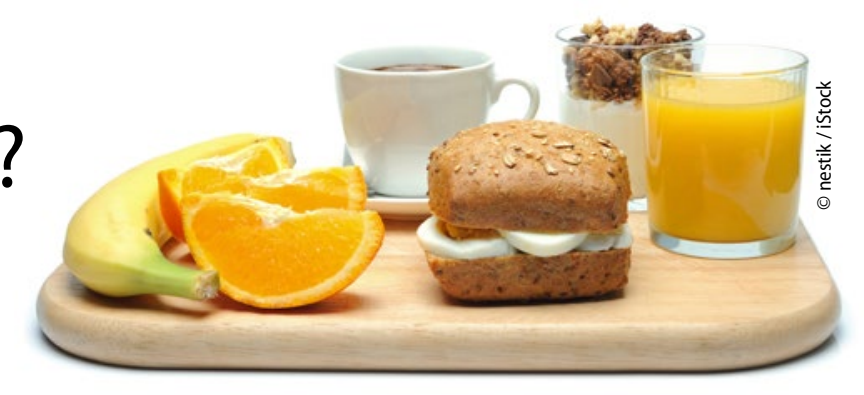

Für die Studie wurden 22 Diabetiker im mittleren Alter von 56,9 Jahren rekrutiert. Ihre Erstdiagnose lag im Mittel 8,4 \pm 0,7 Jahre zurück. Zehn wurden nur mit Diät behandelt, der Rest erhielt zusätzlich Metformin. Sie hatten einen BMI von $28,2 \pm 0,6 \mathrm{~kg} / \mathrm{m}^{2}$ und einen $\mathrm{HbA}_{1 \mathrm{c}}$-Wert von $7,7 \pm 0,1 \%$.

In einem Crossover-Design wurden sie randomisiert auf zwei Tessttage verteilt. Am ersten Testtag wurde morgens, mittags und abends gegessen, am zweiten auf das Frühstück verzichtet. Blut wurde unmittelbar vor den Mahlzeiten und dann 15, 30, 60, 90, 120, 150 und 180 Minuten nach Beginn abgenommen. Am Tag ohne Frühstück wurde morgens der Blutzucker gemessen. Primärer Endpunkt war der Vergleich der postprandialen Glukosewerte nach dem Mittagund dem Abendessen. Daneben wurden weitere Blutwerte bestimmt.

Für alle Vergleichswerte wurde die Fläche unter der Kurve im Zeitraum 0-180 Minuten (AUC 0-180) bestimmt. Nüchternblutzucker, Insulin, C-Peptid, GLP 1, Glukagon und freie Fettsäuren
(FFA) unterschieden sich nicht an den beiden Testtagen ( $p>0,05)$. Am Tag ohne Frühstück lagen nach dem Mittagessen die Plasmaglukose um 36,8\%, die FFA um 41,4\% und das Glukagon um 14,8\% höher als am Tag mit Frühstück ( $p<0,0001$ ), wogegen Insulin um 17\% und GLP 1 um 19\% niedriger lagen.

Die Messungen am Abend ergaben am Tag ohne Frühstück einen um 26,6\% höheren Wert für Glukose. Für FFA waren es $29,6 \%$ und für Glukagon $11,5 \%$. GLP 1 lag 16,5\% unter dem Wert des Frühstückstags, Insulin 7,9\% ( $p=0,001)$. Ohne Frühstück war sowohl mittags als auch abends der Insulin-Peak um 30 Minuten verzögert.

Die Autoren sehen darin eine Demonstration des „Second Meal Phenomenon“, also eine gesteigerten Betazellantwort bei der zweiten Mahlzeit durch Induktion durch eine vorausgegangene Mahlzeit. Es sei auch anzunehmen, dass die GLP-1-Stimulation durch das Frühstück einen anhaltenden Effekt auf die Tagesinsulinsekretion hat. Regelmäßiges Frühstücken bei Typ-2-Diabetes könne eine erfolgreiche Therapie postprandial erhöhter Blutzuckerwerte sein.

- Jakubowicz Det al. Fasting until noon triggers increased postprandial hyperglycemia and impaired insulin response after lunch and dinner in individuals with type 2 diabetes: a randomized clinical trial. Diabetes Care. 2015;38:1820-6

\section{KOMMENTAR}

Diese Untersuchung ist wissenschaftlich interessant. Allerdings wurden nur an zwei Testtagen Daten erhoben, obwohl etwa die Nüchternplasmaglukosekonzentration intraindividuell stark schwankt. Zudem ist das untersuchte Kollektiv heterogen, da etwa die Hälfte der Patienten Metformin nahmen. Die Aussage „ohne Frühstück droht Hyperglykämie" ist daher voreilig. Die Blutzuckerwerte waren nach dem Verzicht aufs Frühstück erwartungsgemäß signifikant geringer, zumindest bis zum Mittagessen. Auch scheint die Lehrmeinung, dass regelmäßiges Frühstücken vor Übergewicht schützt, wissenschaftlich nicht gut belegt [Casazza Ket al. N Engl] Med. 2013;368:446-54]. Abhängig von den Lebensumständen sollten sich Typ-2-Diabetiker daher auch weiter frei zwischen Frühstück und Verzicht entscheiden können.

Dr. med. C. Jaursch-Hancke

\section{Hier steht eine Anzeige.}

\author{
Springer
}

